\title{
The study of the aerodynamic characteristics of dust particles in the air of roadside areas
}

\author{
Anatoly Vasiliev ${ }^{1,1}$, Igor Stefanenko ${ }^{1}$, Valery Azarov ${ }^{1}$ and Denis Nikolenko ${ }^{2}$ \\ ${ }^{1}$ Volgograd State Technical University, 28 Lenin avenue, Volgograd, 400005, Russia \\ ${ }^{2}$ Don State Technical University, 162 Sotcialisticheskaya str., 344022, Russia
}

\begin{abstract}
The article addresses issues, which are related to the determination of aerodynamic characteristics of dust particles in roadside air areas. Was studied dustiness of Volgograd roadside areas.
\end{abstract}

\section{Introduction}

Dust particles, produced by road traffic, considered as an important source of air pollution, since the vehicle fleet is increasing worldwide, therefore, increasing exhaust emissions, as well as particles from tire abrasion and road wear coverings. Emissions without exhaust gases will be important in the future as well, even if the vehicle fleet will be fully electrified [1]. With the exception of emissions directly into the air, particles settle and accumulate together with dust from other sources on road surfaces, forming road dust. Dust is delayed by traffic and / or wind and contributes to air pollution. Sources of road dust strongly differ geographically and in time. Conventional sources are particles of brake and tire wear.

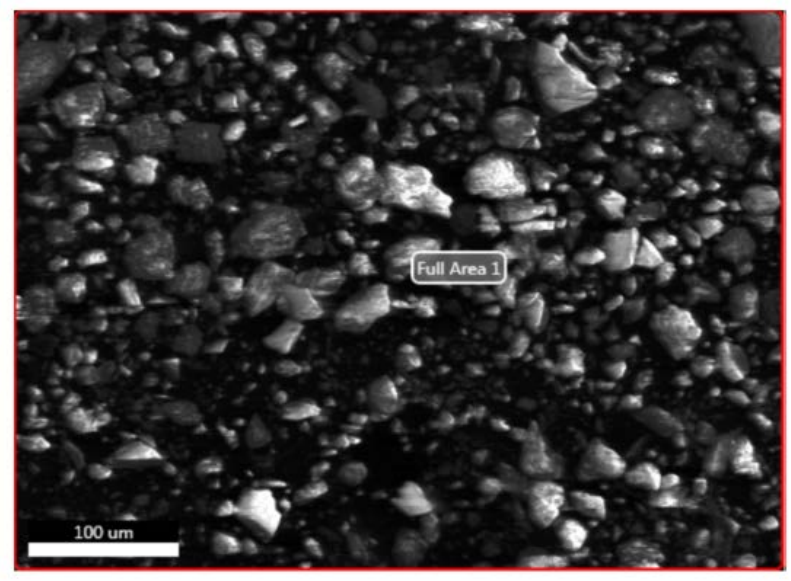

Fig. 1. Micrograph of the investigated road dust [3]

\footnotetext{
${ }^{1}$ Corresponding author: serdariaziev@gmail.com
} 
gravity in a turbulent flow is composed from two processes: a) continuous subsidence of some parts down, inside carrying them pulsating moles, and b) random in direction, frequency and amplitude of particles movement with descents and rises along with carrying them pulsating moles [4]. The sedimentation speed of spherical particles was studied theoretically and experimentally in a wide range of Reynolds numbers. For spherical particles, when calculating the sedimentation speed, there are either theoretical formulas or semi-empirical ratios that describes the experimental data. [5] There are no theoretical models for calculating the sedimentation speed of particles with complex geometric shape (for example, particles of anthropogenic natural dust, dust storms, pollen of plants, powder materials), and therefore, exist several experimental methods.

The first settling process of small suspended particles by gravity in a turbulent flow continuous settling of particles down, despite the pulsating movement of carrier gas moles, is described in the Stokes approximation by the equation,

$$
\mathrm{v}_{\mathrm{g}}=\tau \mathrm{\tau}
$$

Where $\mathrm{g}$ - acceleration of gravity; $\tau$ - particle relaxation time. This expression is derived for the case of free sedimentation of particles in a stationary laminar flow.

The equation of the vertical motion of a Stokes particle in a horizontal flow by gravity, has the form:

$$
\frac{d V_{p}}{d t}+\beta V_{p}=\beta V^{\prime} \sin \omega t-g, V_{p}(0)=0
$$

where vp - settlement speed of particle; $\mathrm{t}$ - considered point of time; $\beta-$ particle inertia factor, sometimes called "Time constant"; $\omega$ - Langrangian ripple frequency; V' amplitude of the transversal component of the speed pulsation of turbulent flow.

It should also be emphasized, that the shape of the particle has a major impact on the aerodynamic properties and on the trajectory of the dust particles movement.

The complexity to determine the sedimentation speed of road dust is also to the fact, that there is a problem in selection of these dust particles.

The authors [6] suggested a method to determine equivalent aerodynamic diameter of aerosol particles with non-spherical shape, when they settle in the field of gravity in the range of Reynolds numbers from 0,1 to 6,0 .

Particles, if they are not too large, react to random turbulent pulsations of the environment and along with translational motion, together with the flow, they make, under their influence, a pulsation (oscillatory movement) relative to carrying them gas moles and random movement along with the moles of gas, called turbulent diffusion of particles.

\section{Methods}

Like the motion of gas moles, the pulsating and diffusive particle movement are random (stochastic) in nature and therefore described statistically. The settling of particles by gravity has the same nature: the particles settle down, making random descents and ascents together with the pulsating moles, carrying them. [4]

The special theory of stationary random processes, or, more precisely, the theory of stationary, received the good interest in practice. Random functions (since the argument of a stationary random function in general case, may not be time).

For a stationary random function $\mathrm{C}(\mathrm{t})$ the correlation function (3) depends not on both its arguments $\mathrm{t} 1$ and $\mathrm{t} 2$, but only on the difference $\Delta \mathrm{t}$ between them, i.e. by interval length [7]: 


$$
\mathrm{K}_{\mathrm{C}}\left(\mathrm{t}_{1}, \mathrm{t}_{2}\right)=\mathrm{K}_{\mathrm{C}}\left(\mathrm{t}_{2}-\mathrm{t}_{1}\right)=\mathrm{K}_{\mathrm{C}}(\Delta \mathrm{t})
$$

where $\mathrm{M}$ - mathematical expectation, $\mathrm{MC}(\mathrm{t} 1)$ and $\mathrm{MC}(\mathrm{t} 2)$ - mathematical expectations of sections, relevant to the fixed values of the arguments $\mathrm{t} 1$ and $\mathrm{t} 2$.

The ordinate distribution density of a random function $\mathrm{f}(\mathrm{C} \mid \mathrm{t})$ and the speed distribution density $\mathrm{f}(\mathrm{C}, \mathrm{v} \mid \mathrm{t})$ are independent from time, where

$$
\mathrm{v}(\mathrm{t})=\frac{\mathrm{dC}(\mathrm{t})}{\mathrm{dt}}
$$

Denote these densities correspondingly $f(C)$ and $f(C, V)$.

Then the average residence time of the stationary random function is higher than given level $\mathrm{C}_{\text {norm }}$ during the time $\mathrm{T}$, he average number of outputs at the same period of time and the average output duration is determined by formulas (4-6).

$$
\begin{aligned}
\overline{\mathrm{t}}=\mathrm{T} \int_{\mathrm{C}_{\text {norm }}}^{\infty} \mathrm{f}(\mathrm{C}) \mathrm{dC} \\
\overline{\mathrm{n}}=\mathrm{T} \int_{0}^{\infty} \mathrm{vf}\left(\mathrm{C}_{\text {norm }}, \mathrm{v}\right) \mathrm{dv} \\
\tau=\frac{\overline{\mathrm{t}}}{\overline{\mathrm{n}}}
\end{aligned}
$$

This method allows to obtain the characteristics of the dispersed dust composition in the air, and to determine the average residence time of the fractional concentration above given level, i.e. to predict the content of small-dispersed dust, depending on various conditions, and to assess the impact on people's health, that live near highways.

\section{Results and Discussion}

Also, the authors of the article conducted studies on the roadside territories of the urban environment of Volgograd. The importance of the urban pollution by small-dispersed particles was highlighted in works [8-11]. For the study, 50 measurement points were selected at various intersections of the city. For measuring was used a manual particle counter HANDHELD 3016 IAQ (made in the USA). The manual particle counter HANDHELD 3016 IAQ is used to measure the mass concentration of aerosol particles of different fractions (PM0.5, PM1, PM2.5, PM5, PM10, TPM, where PM0.5 is the concentration of particles smaller than $0.5 \mu \mathrm{m}, \mathrm{PM} 1$ is the concentration of particles less than $1 \mu \mathrm{m}$ in size, PM2.5 is the concentration of particles smaller than $2.5 \mu \mathrm{m}$, PM5 is the concentration of particles smaller than $5 \mu \mathrm{m}$, PM10 is the concentration of particles smaller than $10 \mu \mathrm{m}$, TPM is the concentration of all fractions of particles / total concentration). 
Table 1. Results of research

\begin{tabular}{|c|c|c|c|c|c|c|c|c|c|c|c|c|}
\hline \multirow{3}{*}{ № п/п } & \multicolumn{12}{|c|}{ Time of the research } \\
\hline & \multicolumn{6}{|c|}{ Morning time } & \multicolumn{6}{|c|}{ Evening time } \\
\hline & PM 0.5 & PM 1 & PM 2.5 & PM 5 & PM 10 & TPM & PM 0.5 & PM 1 & PM 2.5 & PM 5 & PM 10 & TPM \\
\hline 1 & 14.74 & 23.81 & 34.69 & 56.76 & 43.56 & 51.46 & 8.41 & 16.7 & 31.55 & 62.34 & 82.04 & 87.12 \\
\hline 2 & 16.78 & 21.45 & 29.45 & 38.34 & 58.45 & 74.23 & 11.84 & 21.78 & 36.4 & 56.37 & 70.6 & 116.37 \\
\hline 3 & 12.65 & 23.03 & 30.29 & 38.95 & 45.58 & 49.74 & 16.6 & 30.93 & 41.2 & 53.09 & 64.6 & 81.24 \\
\hline 4 & 11.23 & 17.65 & 27.82 & 37.65 & 40.02 & 41.26 & 9.58 & 17.9 & 32.36 & 99.79 & 153.42 & 170.52 \\
\hline 5 & 10.25 & 20.74 & 34.76 & 65.08 & 86.53 & 103.64 & 9.28 & 16.36 & 24.01 & 43.05 & 56.12 & 57.97 \\
\hline 6 & 17.05 & 35.1 & 54.09 & 69.91 & 74.59 & 79.21 & 21.89 & 41.53 & 53.74 & 64.02 & 74.94 & 79.1 \\
\hline 7 & 13.28 & 24.00 & 30.34 & 36.66 & 41.34 & 43.19 & 23.6 & 47.22 & 62.58 & 73.24 & 80.06 & 83.76 \\
\hline 8 & 12.79 & 22.91 & 28.94 & 33.27 & 37.36 & 38.29 & 15.71 & 28.75 & 36.85 & 44.48 & 48.18 & 52.81 \\
\hline 9 & 13.62 & 24.35 & 31.05 & 41.87 & 61.38 & 74.78 & 19.42 & 40.26 & 60.59 & 76.46 & 89.72 & 100.82 \\
\hline 10 & 13.16 & 23.75 & 30.28 & 40.88 & 49.86 & 52.63 & 21.51 & 40.99 & 55.03 & 71.22 & 84.29 & 94.92 \\
\hline 11 & 73 & 17.79 & 30.53 & 55.05 & 73.77 & 77.01 & 6.71 & 13.05 & 26.07 & 79.97 & 24.59 & 97.37 \\
\hline 12 & 12.31 & 22.06 & 28.98 & 40.97 & 54.43 & 59.98 & 16.38 & 29.23 & 38.7 & 51.65 & 63.15 & 71.94 \\
\hline 13 & 8.83 & 17.47 & 29.08 & 55.67 & 73.61 & 78.24 & 8.05 & 16.14 & 30.12 & 60.74 & 79.27 & 82.5 \\
\hline 14 & 13.73 & 25.42 & 35.23 & 62.41 & 94.98 & 107.92 & 14.86 & 26.59 & 35.28 & 44.79 & 53.37 & 59.84 \\
\hline 15 & 13.09 & 23.36 & 31.26 & 46.83 & 66.33 & 85.75 & 20.91 & 40.34 & 59.95 & 91.18 & 123.36 & 157.1 \\
\hline 16 & 12.84 & 22.92 & 29.19 & 34.61 & 37.53 & 39.38 & 18.58 & 35.22 & 46.31 & 55.23 & 60.11 & 64.73 \\
\hline 17 & 7.7 & 15.56 & 28.42 & 58.55 & 87.99 & 102.32 & 6.67 & 12.17 & 18.92 & 35.61 & 48.49 & 49.87 \\
\hline 18 & 7.34 & 14.88 & 25.81 & 50.41 & 68.35 & 71.59 & 7.89 & 14.56 & 23.45 & 81.34 & 25.67 & 102.34 \\
\hline 19 & 14.81 & 27.56 & 37.18 & 66.83 & 124.75 & 142.31 & 17.75 & 31.95 & 40.57 & 81.52 & 124.43 & 131.82 \\
\hline 20 & 8.55 & 15.91 & 26.22 & 54.11 & 74.2 & 78.82 & 9.12 & 15.17 & 38.65 & 55.62 & 84.37 & 82.9 \\
\hline 21 & 13.39 & 23.77 & 29.94 & 36.97 & 43.78 & 54.88 & 15.49 & 28.36 & 38.12 & 48.38 & 56.76 & 79.42 \\
\hline 22 & 12.95 & 23.6 & 31.14 & 45.52 & 76.43 & 102.32 & 21.52 & 38.85 & 51.82 & 74.93 & 111.01 & 182.66 \\
\hline 23 & 14.25 & 26.8 & 36.17 & 59.67 & 102.77 & 142.53 & 17.29 & 32.08 & 41.89 & 52.45 & 62.59 & 76.46 \\
\hline 24 & 5.97 & 11.98 & 18.92 & 40.03 & 54.86 & 57.17 & 5.22 & 10.12 & 16.58 & 36.03 & 52.03 & 57.57 \\
\hline 25 & 13.04 & 23.35 & 29.93 & 37.46 & 48.96 & 55.9 & 12.43 & 21.56 & 43.76 & 69.87 & 85.32 & 86.78 \\
\hline 26 & 14.09 & 24.57 & 30.72 & 41.32 & 57.12 & 65.9 & 16.92 & 31.59 & 42.84 & 56.64 & 70.88 & 84.28 \\
\hline 27 & 8.01 & 14.95 & 25.04 & 46.56 & 61.58 & 62.51 & 13.89 & 24.85 & 43.35 & 81.06 & 106.61 & 115.85 \\
\hline 28 & 12.46 & 22.22 & 27.44 & 33.21 & 40.43 & 47.36 & 14.97 & 27.25 & 35.58 & 44.77 & 51.99 & 60.31 \\
\hline 29 & 12.78 & 22.67 & 29.88 & 50.72 & 94.21 & 125.64 & 19.23 & 35.05 & 47.84 & 63.83 & 79.04 & 97.07 \\
\hline 30 & 16.98 & 44.24 & 90.83 & 148.14 & 194.56 & 222.76 & 7.23 & 15.52 & 35.14 & 66.61 & 81.43 & 83.74 \\
\hline 31 & 12.04 & 21.56 & 26.88 & 32.83 & 38.68 & 43.3 & 15.85 & 27.71 & 35.52 & 44.03 & 51.24 & 61.41 \\
\hline 32 & 13.06 & 23.64 & 30.37 & 40.61 & 45.29 & 47.14 & 19.16 & 35 & 45.84 & 56.56 & 68.26 & 81.67 \\
\hline 33 & 14.54 & 35.8 & 57.57 & 84.82 & 104.52 & 110.99 & 8.38 & 15.13 & 26.07 & 52.37 & 70.7 & 77.67 \\
\hline 34 & 16.03 & 29.89 & 38.91 & 52.88 & 70.82 & 24.69 & 13.1 & 24.13 & 32.33 & 42.6 & 53.71 & 60.18 \\
\hline 35 & 11.57 & 21.58 & 36.83 & 87.05 & 122.74 & 154.64 & 4.94 & 9.51 & 16.74 & 40.09 & 56.08 & 59.78 \\
\hline 36 & 9.54 & 17.98 & 32.65 & 71.75 & 105.69 & 123.71 & 5.18 & 9.86 & 16.63 & 36.72 & 47.25 & 56.49 \\
\hline 37 & 8.11 & 15.15 & 28.59 & 74.98 & 115.59 & 137.17 & 4.23 & 7.98 & 13.3 & 30.3 & 42 & 48.47 \\
\hline 38 & 7.6 & 14 & 25.59 & 61.52 & 93.31 & 105.79 & 4.88 & 9.73 & 17.61 & 40.67 & 54.52 & 57.76 \\
\hline 39 & 7.73 & 14.16 & 27.51 & 72.41 & 108.1 & 125.66 & 5.16 & 9.44 & 15.69 & 35.32 & 49.36 & 56.75 \\
\hline 40 & 5.95 & 11.74 & 23.78 & 63.35 & 96.7 & 106.87 & 5.52 & 1065 & 19.03 & 44.19 & 59.6 & 61.45 \\
\hline 41 & 8.01 & 14.67 & 25.16 & 56.61 & 79.13 & 86.53 & 5.13 & 10.45 & 19.49 & 47.84 & 65.98 & 68.75 \\
\hline 42 & 10.38 & 18.04 & 26.25 & 42.14 & 57.94 & 62.66 & 7.56 & 13.62 & 28.72 & 49.87 & 76.7 & 84.24 \\
\hline 43 & 14.28 & 23.11 & 30.6 & 43.3 & 60.66 & 65.74 & 5.83 & 11.41 & 20.32 & 46.28 & 65 & 67.31 \\
\hline 44 & 8.99 & 15.9 & 22.92 & 37.47 & 48.59 & 49.98 & 6.18 & 12.54 & 23.12 & 52.34 & 68.76 & 72.34 \\
\hline 45 & 7.33 & 12.84 & 19.27 & 35.87 & 50.89 & 57.82 & 5.62 & 11.73 & 22.6 & 54.19 & 72.72 & 88.9 \\
\hline 46 & 7.48 & 13.98 & 23.48 & 48.76 & 87.38 & 104.94 & 4.97 & 10.82 & 21.58 & 57.81 & 90.57 & 100.74 \\
\hline 47 & 9.23 & 16.23 & 25.58 & 44.86 & 60.85 & 65.94 & 7.18 & 14.44 & 26.41 & 57.52 & 83.46 & 92.24 \\
\hline 48 & 6.66 & 12.74 & 21.08 & 39.04 & 66.28 & 66.82 & 5.13 & 11.2 & 20.36 & 46.35 & 68.97 & 77.75 \\
\hline 49 & 11.65 & 20.7 & 26.35 & 35.2 & 50.02 & 57.42 & 12.69 & 18.56 & 23.75 & 44.72 & 59.36 & 63.63 \\
\hline 50 & 15.62 & 27 & 34.89 & 47.89 & 55.03 & 57.23 & 15.4 & 25.43 & 34.35 & 50.32 & 75.34 & 23.76 \\
\hline
\end{tabular}




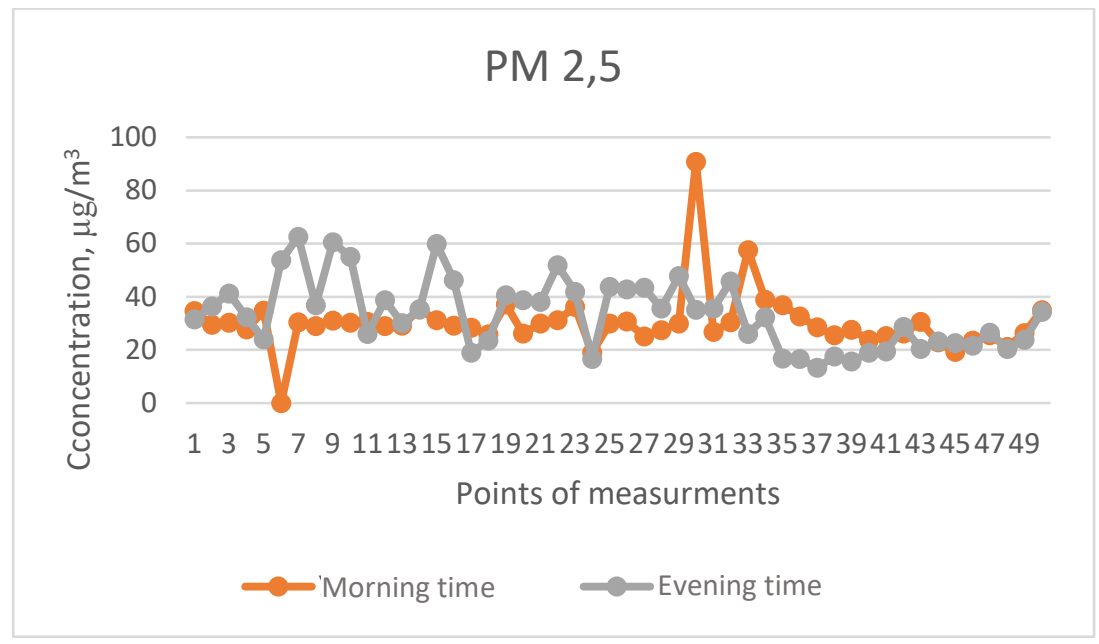

Fig. 2. The distribution of concentrations of PM2.5 fraction in the morning and evening at measurement points $1-50$

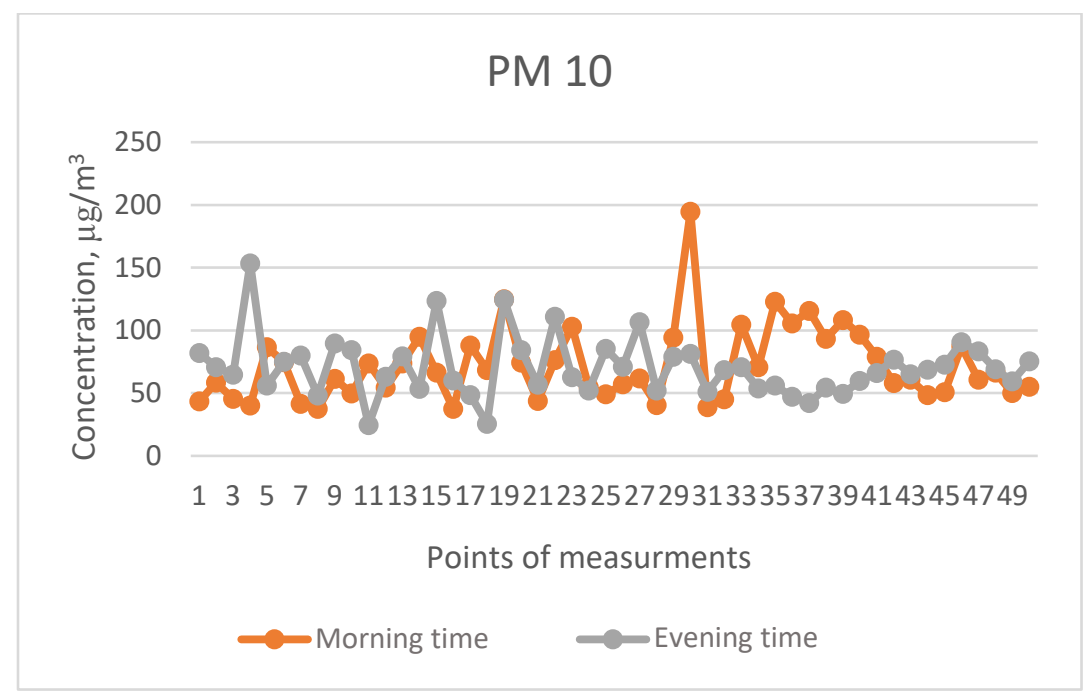

Fig. 3. The distribution of concentrations of the PM10 fraction in the morning and evening at measurement points $1-50$ 


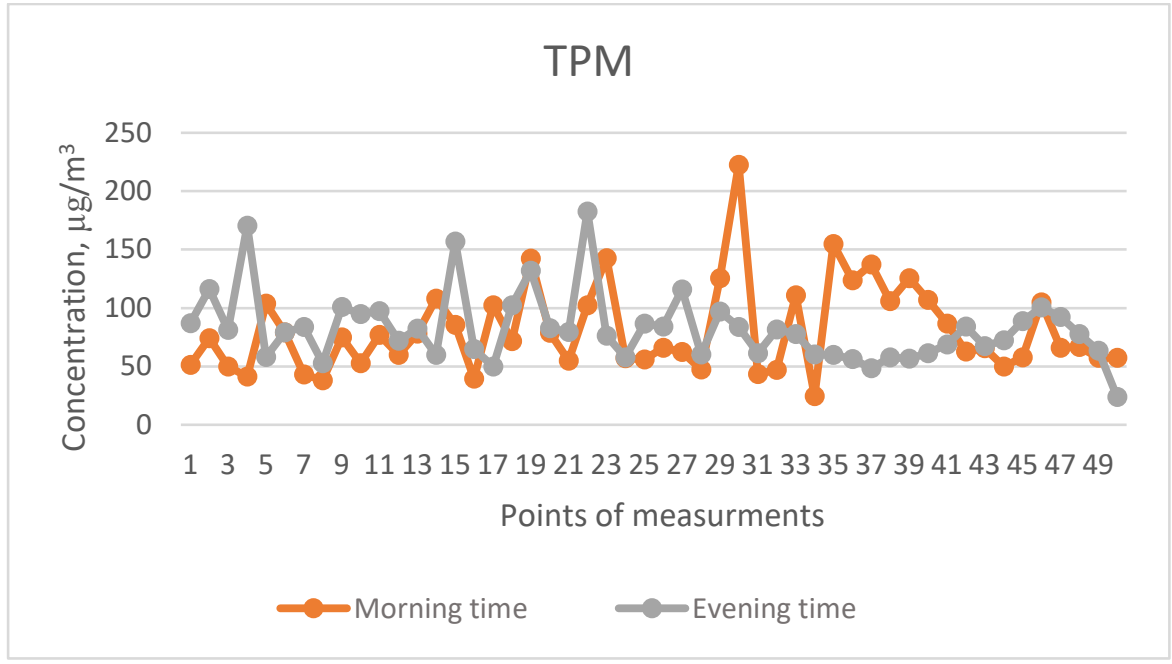

Fig. 4. The distribution of concentrations of the PM10 fraction in the morning and evening at measurement points $1-50$

\section{Summary}

Hence. it may be concluded. that that in the evening there is an excess of the concentrations of PM0.5. PM1. PM2.5. PM5. PM10. TPM over the morning. The PM2.5 concentrations are in range from 13.3 to $62.58 \mu \mathrm{g} / \mathrm{m} 3$. the PM10 concentrations are in range from 24.59 to $194.56 \mu \mathrm{g} / \mathrm{m} 3$. and TPM - from 38.29 to $222.76 \mu \mathrm{g} / \mathrm{m} 3$.

It should be noted. that with an increase of the transport speed movement. the proportion of large particles. which wasn't exist at low intensity. increases spasmodically. it is possibly due to the appearance of turbulence of air masses at the intersection borders. and due to significant centrifugal forces that contribute to the separation of large particles on the periphery of the air vortex.

These results lead to the conclusion. that the distribution of dust particles in the roadside territories of Volgograd is heterogeneous. but significant excesses are observed at public transport stops and at major road junctions in the city. It could be noticed. that in the evening the concentration of dust particles much higher than the morning measurements. which is justified by the traffic congestion on the roads.

The task of reducing the negative impact of the transport and road complex on the environment is very important. since the faster growth of the vehicle fleet in comparison with the development of public roads. leads to an increase in their traffic congestion. lower average speed and. as a result. to deterioration of the environment due to pollution by harmful emissions.

\section{References}

1. V.R.J.H. Timmers. P.A.J. Achten. Non-exhaust PM emissions from electric ve-hicles. Atmos. Environ. 134, 10-17 (2016) . https://doi.org/10.1016/j.atmosenv.2016.03.017.

2. N.S. Barikaeva. D.A. Nikolenko. Yu.P. Ivanova. About Forecasting Air Pollution in the Construction of Highways, International Multi-Conference on Industrial Engineering and Modern technologies IOP Conf. Series: Materials Science and 
Engineering. $\quad$ 463, 042016, (2018). URL: https://iopscience.iop.org/article/10.1088/1757-899X/463/4/042016/pdf.

3. V.N. Azarov. I.V. Stefanenko. N.Yu. Karapuzova. D.A. Nikolenko. E.Yu. Kozlovtseva. Monitoring of Fine Dust Pollution of Urban Air Nearby Highways. International Review of Mechanical Engineering (I.RE.M.E.). 8(12). pp. 657-662 (2018)

4. E.P. Mednikov, Turbulent transport and deposition of aerosols (M.: Science. 1981)

5. I.V. Kabaeva. A.G. Shestakov. A.S. Artyukhin, About the distribution and sedimation of dust particles up to $10 \mathrm{mkm}$ in a horizontal turbulent flow, (2006)

6. V.L. Istomin. K.P. Kutsenogiy, Method for determining the aerodynamic diameter of aerosol particles of complex geometric shape in the range of Reynolds numbers from 0.1 to 6.0

7. A.A. Sveshnikov. Applied methods of the theory of random functions (Textbook. allowance. SPb) Doe. 2011.252 s.

8. V.N. Azarov. N.M. Sergina. T. Kondratenko, Problems of protection of urban ambient air pollution from industrial dust emissions, MATEC Web of Conferences. : International Science Conference SPbWOSCE-2016 "SMART City", 106, (2017). URL: $\quad$ https://www.matecconferences.org/articles/matecconf/abs/2017/20/contents/contents.html.

9. V.N. Azarov. A.A. Sakharova. V.V. Lupinogin. A.I. Evtushenko. M.A. Nikolenko Analysis of the chemical composition of dust particles in the warehouse of building materials, IOP Conference Series: Materials Science and Engineering. Chemical. Ecological and Power Engineering. - Publishing Ltd. v. 537, 7 (2019). - URL: https://iopscience.iop.org/article/10.1088/1757-899X/537/6/062058/pdf.

10. I.V. Stefanenko. V.N. Azarov. D.P. Borovkov. Experimental Optimization of Dust Collecting Equipment Parameters of Counter Swirling Flow with Coaxial Leadthrough for Air Ventilation System and Dust Elimination, All-Russian research-to-practice conference "Ecology and safety in the technosphere: current problems and solutions" IOP. 224(1), 9 (2019). - URL: https://iopscience.iop.org/article/10.1088/17551315/224/1/012037/pdf.

11. R.A. Burkhanova. I.A. Kovtunov. V.N. Azarov. Investigation of the Parameters of Discarded Dust in the Manufacture of Products from Chrysotile Asbestos and Cement, IOP Conference Series: Earth and Environmental Science. International science and technology conference "Earth science", 272, 8 (2019). URL: https://iopscience.iop.org/article/10.1088/1755-1315/272/2/022150/pdf (Published online: 21 June 2019). 